\title{
Complete title: Low Voltage Zones as the Atrial Fibrillation Substrates: Relationship with Atrial Fibrillation Initiation, Perpetuation, and Termination.
}

\author{
Zheng Liu ${ }^{1}$, Yuanfeng $\mathrm{Gao}^{2}$, xiandong yin ${ }^{1}, \mathrm{Yu} \mathrm{Xia}^{3}$, Chang-yan Guo ${ }^{4}$, Yuxing Wang ${ }^{2}$, liu \\ xiaoqing ${ }^{5}$, xingpeng $\mathrm{Liu}^{5}$, Pihua Fang ${ }^{6}$, and Xinchun Yang ${ }^{1}$ \\ ${ }^{1}$ Beijing Chao-yang Hospital, Capital Medical University, Beijing \\ ${ }^{2}$ Beijing Chaoyang Hospital \\ ${ }^{3}$ State Key Laboratory of Cardiovascular Disease, Cardiac Arrhythmia Center, Fuwai \\ Hospital, National Center for Cardiovascular Diseases, Chinese Academy of Medical \\ Sciences and Peking Union Medical College \\ ${ }^{4}$ Xilin gol league central hospital \\ ${ }^{5}$ Beijing Chao-Yang Hospital \\ ${ }^{6}$ State Key Laboratory of Cardiovascular Disease, Fuwai Hospital, National Center for \\ Cardiovascular Diseases, Chinese Academy of Medical Sciences and Peking Union Medical \\ College, Beijing, People's Republic of China.
}

May 21, 2020

\begin{abstract}
Background: Low voltage Zones (LVZ) were usually targeted for ablation in atrial fibrillation (AF). But its relationship with AF initiation, maintenance, and termination remains to be studied. We tried to explore the relationships. Methods and Results: Consecutive AF patients were enrolled for assessment AF inducibility, AF duration or AF termination before ablation. Inducible AF was defined if induced AF last over 30 seconds. Sustainable AF was defined if it last over 300 seconds. Terminable AF was defined if it could be cardioverted into sinus rhythm within 1-hour after ibutilide administration. Voltage mapping was performed in sinus rhythm for all patients before stimulation or after cardioversion. LVZ was quantified as the percentage of LVZ area(LVZ\%)to left atrium (LA) body surface. A total of 86 patients enrolled for AF induction and 36 for AF termination. $32(37.2 \%)$ patients had inducible AF, $24(27.9 \%)$ were sustainable, and $12(33.3 \%)$ were terminable. Inducible AF patients had higher $\mathrm{LVZ} \%$ in anterior wall $(18.6 \pm 24.6$ vs. $7.0 \pm 12.1, \mathrm{P}=0.014)$. Global $\mathrm{LVZ} \%$ was not different between inducible and uninducible AF patients. Global LVZ\% was higher in patients with sustainable AF or interminable AF (LVZ\%: sustainable vs unsustainable $\mathrm{AF}: 10.6 \pm 12.1$ vs. $0.8 \pm 0.8, \mathrm{p}=0.001$; terminable vs. interminable: $17.1 \pm 13.5$ vs.40.6 $\pm 24.5, \mathrm{p}<0.001$ ). Sustainable AF had larger LVZ\% in roof, anterior wall, septum, floor. Interminable AF patients had higher LVZ\% in anterior wall, septum, posterior wall and floor. Higher LVZ\% was independent risk factor of recurrence $(\mathrm{OR}=1.015, \mathrm{P}=0.042)$. Conclusion: The association between LVZ with AF initiation, perpetuation and termination were different.
\end{abstract}

\section{Hosted file}

2020-5.pdf available at https://authorea.com/users/324941/articles/453010-complete-titlelow-voltage-zones-as-the-atrial-fibrillation-substrates-relationship-with-atrialfibrillation-initiation-perpetuation-and-termination 